\title{
Optimal Coverage and Connectivity Control Based on Associated Target in WSN
}

\author{
Yunxing Shu ${ }^{1}$ Zeyu $\operatorname{Sun}^{12}$ and Ge bo ${ }^{1 *}$ \\ 1. Computer and Information Engineering, Luoyang Institute of Science and \\ Technology, Luoyang 471023, China. \\ 2. Electrical and information Engineering, Xi'an Jiaotong University Xi'an \\ 710049, China \\ jsjsdz@163.com
}

\begin{abstract}
Coverage under certain conditions, with minimum sensor nodes to complete the coverage area and connectivity in wireless sensor networks has become a challenging one with the core issues. To this end, we propose a target associated with optimal coverage and connectivity control method using a double square to the target node within the planning area within the square, correlation model obtained by the sensor node and the destination node, for the entire coverage area is the use of probability determined to meet the minimum expectations of sensor nodes under cover conditions, and gives a probability model and the reasoning process connectivity square area outside edge node. Experimental results show that the error theoretical values with the simulation results of the method is less than $7.02 \%$, to verify the effectiveness of the algorithm, reducing the allocation of network resources, you can better assess the performance of network coverage and connectivity.
\end{abstract}

Keywords: wireless sensor networks, coverage and connectivity, associated target, sensor nodes

\section{Introduction}

Wireless sensor networks (Wireless Sensor Network WSN) is composed of a large number of cheap have perception, computing and communication capabilities of sensor nodes through the network formed by self-organization, widely used in various fields of science defense, medical, exploration, and environmental monitoring.

Coverage and connectivity issues have become one of the fundamental problems in wireless sensor network research. Sensor node deployment is generally divided into two kinds of deterministic deployment and random deployment. For deterministic deployment, which is mainly research on two-dimensional and three-dimensional space is optimized node deployment strategy to artificial means to achieve the requirements of different network coverage and connectivity [1]. In a large number of sensor nodes or network deployment area is not suitable for manual deployment, usually in a random manner to deploy the network. In addition, most applications do not require sensor network remains completely covered state, as long as the sensor network node for monitoring the work area to maintain a reasonable coverage ratio, you can complete some work. Therefore, coverage and connectivity issues randomly deployed wireless sensor networks is very important. How to meet certain coverage under conditions of complete sensor nodes with minimal coverage and connectivity of the specified area is a challenging problem classes. Based on the above ideas, their resolve three issues: first, the target node monitoring plan to within a square area, the establishment of a sensor node and the destination node associated with the model, so that the target of concern in the sensing range of multiple 
sensor nodes inside. Second, for the entire monitoring area side length, radius and the required sensor node coverage, calculated using probabilistic expectations minimum sensor nodes under cover conditions satisfied. Third, to ensure balance and meet the scheduling node status under the premise of quality network coverage, taking into account the actual situation given the existence of an edge node connectivity probability model, calculated so as to achieve the target area within the scope of the monitoring results more precise purpose. In this paper, the coverage area of the side length of the calculation and reasoning, given the different rates of coverage and connectivity to deploy the required number of value nodes.

\section{Background}

In recent years, many experts at home and abroad on various characteristics of wireless sensor networks a lot careful and thorough research. [6] proposed a maintain connectivity covering algorithm using relative adjacency diagrams and deployment characteristics given node itself deploying sensor nodes in a mobile network in communication with the process to maintain coverage. topology of the algorithm is to select an arbitrary sensor node moving direction are formed to maintain the network connectivity, coverage characteristics of a mobile node depending on the target network connectivity constraints the algorithm, although to some extent can maintain connectivity coverage of the network, but the effective coverage area is mainly reflected in the locality. for global terms, when accumulated to a certain number of the destination node, its coverage will show a gradual downward trend evident, the algorithm has some limitations of the literature [7] proposed a centralized cluster configuration protocol covering $K$ the literature on the use of the network covered by the agreement to build the model for analysis and shows the four $K$ covering configuration protocol, namely : Centralized Randomized Connected $K$ Coverage (CERAC); $T$-Clustered Randomized Connected $K$-Coverage ( $T$-CRACCk); $D$ Clustered Randomized Connected $K$-Coverage $(D$-CRACCk) and Distributed randomized Connected $K$-Coverage (DIRACCk). this proved that when $K \geqslant 3$ literature, the network model is formed after covering the Relax triangle of side length sensing radius $r$, at least one sensor node $K$; communication, and the radius of a given radius when finished sensing coverage $K$ Compare the relationship, but also gives the formula for calculating the density of sensor nodes required to be completed within the area covered by the minimum number of sensor nodes in the calculation process and the monitoring area. Although the research results achieved using the least effective sensor nodes within the monitoring area of the target node cover, but the higher complexity of the algorithm to calculate the amount of sensor nodes deployed relatively harsh conditions. [8] proposed to maximize the network lifetime $K$ of the discrete barrier covering algorithm this algorithm from the theoretical analysis of the specific network Model disorders and conditions of the upper limit of the coverage exists, the mobile target node concerned completed effectively cover; energy consumption in the network, the sensor node gives a scheduling algorithm, which is introduced to the sensor nodes greedy algorithm redeployment, use scheduling mechanism between sensor nodes to complete the state transition between sensor nodes, and ultimately achieve the aim of maximizing the network lifecycle. the algorithm when moving target node of interest coverage, the need to ensure the distribution of the monitoring sensor nodes remain connected to the area or intersection, once a moving target node appears in coverage "blind spot" when it can not complete the effective coverage of a moving target nodes, the network model is too idealistic. [9] are given by way of a mixed integer programming a heuristic covering algorithm the algorithm of the sensor nodes and the sensor node set intersection heuristic adjustable sensing radius covered the large amount of calculation algorithm, adjustable poor, in order to further improve the literature[9]algorithm, Yang S H[10]tin the coverage area of 
the target node approximation seen as the monitoring area, the use of high-density deployment of sensor nodes to build a collection of complete coverage connectivity coverage of the target coverage area in the research literature on the basis of the two, Liu $\mathrm{H}$ et al [11] on the target coverage problem a lot of research work in the sensor node can define a coverage condition of a target node, the target node gives a coverage solving process at any time. Thanks limited conditions, making the monitoring area coverage applied more narrowly, do not apply to the actual coverage scenarios. literature [12] through artificial bee colony algorithm and particle swarm optimization scheduling problem for sensor nodes are optimized configuration through heuristic find the best deployment path, the calculation method of the sensor node position calculation and theoretical upper limit of the life cycle of the network after the optimum deployment. [13] proposed an energy efficient routing algorithm to maintain connectivity coverage, An Energy-Efficient Coverage and Connectivity Preserving Routing Algorithm (ECCRA), the algorithm uses the network model to construct the double coverage area, covered by calculating the probability of relevant knowledge and coverage probability value expectations, and ultimately determine the ceiling covered with expectations and effective monitoring area for complete coverage of the minimum sensor Number of nodes in the literature [12] and [13], to some extent, have been completed in the effective coverage of the monitored area, to suppress a rapid energy consumption of sensor nodes, but the calculation of the two algorithms is large, a high complexity of the algorithm, the nodes completion of the monitoring area covered by the longer time.

\section{Problem Definition and Network Models}

\subsection{Basic Definition}

Coverage and connectivity studied in this paper is based on the fundamental assumptions: Assumption 1: coverage radius and communication radius of each node are equal and are showing a disc-shaped, and the mobile nodes are synchronized parallel. Assumption 2: Get through some own node localization algorithm specific location information. Hypothesis 3: All sensor nodes are uniformly randomly deployed in side length 1 of the square within the monitoring area $\Omega$, consider the deployment of border regions factors.

Definition 1: Disposed two-dimensional plane $E$, the node coordinates $s_{i}\left(x_{i}, y_{i}\right)$, the coordinates of the target $t_{k}\left(x_{k}, y_{k}\right)$, Euclidean distance between the nodes is less than the $s_{i}$ and $s_{i} t_{k}$ perception radius $r_{s}$, namely: is said to be the point $t_{k} s_{i}$ covered.

Definition 2:Let $S$ be a collection of $n$ nodes randomly distributed in the target area, that is $S=\left\{s_{1}, s_{2}, s_{3} \ldots s_{n}\right\}, E$, is the set of edges of the network graph, which represent the set of edge whose $e_{i j}=1$, where $e_{i j}$ denotes the positional relationship of node $s_{i}$ and target node $p_{j}$, and $e_{i j}=1$ if and only if the Euclidean distance of the two nodes is less than or equal to the sensing radius $r_{s}$, otherwise $e_{i j}=0 . W=\left\{w_{1}, w_{2}, w_{3} \ldots w_{N}\right\}$ is a set of initial energy of the sensor nodes, $W$ follows $W \sim N\left(u, \sigma^{2}\right)$ normal distribution, $w_{i}$ represents the initial energy of the sensor nodes $s_{i}, w_{i}$ is the maximum energy amount during the working process of node $s_{i}$.

Definition 3: Given a target set $T=\left\{t_{1}, t_{2}, t_{3} \ldots t_{k}\right\}$ and the sensor node set $\mathrm{V}=\left\{v_{1}, v_{2}\right.$, $\left.v_{3} \ldots v_{n}\right\}$, in a time slot, if any one of the target set $t_{k}$ target by at least a set of nodes within a node $\mathrm{V}$ is covered, called the target set $T$ full coverage.

Definition 4: set the node $s_{i}, s_{j}$ they cover the target area for $C_{i}$ and $C_{j}$, and $C_{i} \cap C_{j} \neq \varnothing$ is said to node $s_{i}, s_{j}$ covering association.

Definition 5:A non-wireless sensor network to the communication diagram $G=(V, E)$ as a model, where $V$ is the set of sensor nodes, and $|V|=n$; if and only if the node $\mathrm{p}$ and node $q$ within the communication range of each other, the claimed $E$ memory in an 
undirected edge $(p, q)$; number of neighbor nodes of the node $p$ is called $p$, expressed as $d$ $(p)$, represents the number of communication nodes can be performed with other nodes it; communication graph $G d_{\text {min }}(G)=\min _{\forall p \in V}\{d(p)\}$ is expressed as the lowest node.

Definition 6: When the communication graph $G$ where any pair of nodes in a communication path exists, saying the network is connected, otherwise it's not connected. For a connected network, all of its nodes are able to hop or multi-hop way to communicate. On the other hand, in the communication network will be an isolated subnetwork, there are some which are formed a number of sub-network nodes communicate with each other, but can not communicate with each other between the various subnet works.

\subsection{Establishment and Variable Parameters Described}

Table 1. Parameter Description Establishment and Variable

\begin{tabular}{cc}
\hline Parameters & Variable Description \\
\hline$l$ & coverage area of a square side length \\
$\Omega$ & network areas, $\Omega=l^{2}$ \\
$n$ & umber of nodes randomly deployed network \\
$r_{s}$ & sensor nodes sensing radius, $r_{s}<l$ \\
$r_{t}$ & sensor nodes communication radius, $r_{t}<l$ \\
$E(C)$ & sensor nodes covering area of expectations \\
$P\left(S_{n}\right)$ & random deploy network of $n$ nodes desired coverage \\
$P\left(C_{n}\right)$ & Random deployment of network connectivity \\
\hline
\end{tabular}

\subsection{Network Model}

In general, the degree of coverage of the target is directly reflected concern, causing the destination node has a higher area coverage concerned, taking into account the relationship between the function of the node $\mathrm{p}$ in the region of the sensor at the desired value with the coverage area $I I$, as shown in Figure 1:

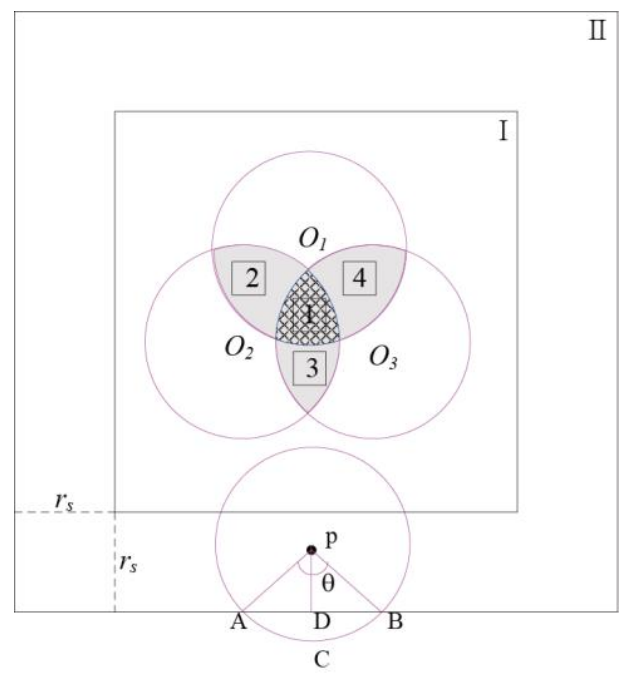

Figure 1. Associated Monitoring Regional Targets Cover Model

Figure 1 shows a model of the target coverage area associated with the monitoring target node 1 to 4 , three sensor nodes within the region $I$, other sensor nodes and the bit 
is the area between regions $I$ and $I I$. Can be seen from Figure 1, the destination node are respectively covered by the sensor nodes, the sensor node and the destination node frequently associated with a value of 2,3 , 4 , i.e. the destination node is currently in the coverage of $k=2$, a double, and a destination node, the sensor node in the three among, the frequent value of 3 .

\section{Problem Definition and Network Models}

\subsection{Network Coverage}

Figure 1 for example is to be analyzed, the square region is divided into two partial areas $l$ and regional $I$ and $I I$. Random deploying nodes in the monitoring area, and these nodes configured as a finite set $S$, the covering area of each node as $E(C)$, so that the probability of each node is covered by $E(C) / \Omega$, when the set of nodes empty, n node network coverage is obtained deployed $P(S)=(1-E(C) / \Omega) \mathrm{n}$ was thus obtained the network node is not the empty set $S$ collectively in the case where the coverage probability values:

$$
P(S)=1-(1-E(C) / \Omega)^{n}
$$

When the number of nodes, which indicates the number of nodes, is sufficiently large, the coverage area is completely covered. Taking into account the influence of boundary conditions, solving node coverage area and the expectations from the square area is divided into regions and regional $I$ and $I I$, therefore, according to the concept of probability expectations, you can get coverage expectations of network nodes:

$$
E(C)=P\left(\Omega_{I}\right) E\left(C_{\Omega_{I}}\right)+P\left(\Omega_{I I}\right) E\left(C_{\Omega_{I I}}\right)
$$

Where $P\left(\Omega_{I}\right)$ and $P\left(\Omega_{I I}\right)$ denote the value of the node is the probability of randomly deployed at regional and regional I and II Lane $E\left(C_{\Omega_{I}}\right)$ and $E\left(C_{\Omega_{I I}}\right)$ represent the expected value of the corresponding cover. Since the deployment of the sensor node to obey a uniform distribution function, thereby obtaining:

$$
\left\{\begin{array}{l}
P\left(\Omega_{I}\right)=\left(l-2 r_{s}\right)^{2} / l^{2} \\
P\left(\Omega_{I I}\right)=4 r_{s}\left(l-r_{s}\right) / l^{2}
\end{array}\right.
$$

Assumed that the node $p$ in the region of the inner $I$, its coverage is completely contained, it is the expected value to obtain coverage:

$$
E\left(C_{\Omega_{I}}\right)=\pi r_{s}^{2}
$$

When the node $p$ in the region of $I I$, its area should be equal to the sensing area of the circumferential area of $S_{A C B D}$ subtracting the arch region. $A$ and $B$ is the intersection of the peripheral sensor nodes $p$ and network boundaries, which is a node $\mathrm{p}$ and the angle $\theta$, the central angle formed by the points $\mathrm{A}$ and $\mathrm{B}$, i.e. $\angle A p B=\theta$, the angle between the base thus obtained:

$$
\begin{aligned}
E\left(C_{\Omega_{I I}}\right) & =4 \int_{0}^{l-r_{s}} \frac{1}{2} r_{s}^{2}(2 \pi-\theta+\sin \theta) d x \int_{0}^{r_{s}} \frac{1}{4\left(l-r_{s}\right) r_{s}} d y \\
& =\frac{r_{s}}{2\left(l-r_{s}\right)} \int_{0}^{l-r_{s}}(2 \pi-\theta+\sin \theta) d x \int_{0}^{r_{s}} d y
\end{aligned}
$$


Theorem 1: Assuming the radius $r_{s}$ given sensor nodes uniformly distributed in a square of side length 1 of the region, in consideration of the boundary elements, the expected value of the node coverage:

$$
E(C)=\left(\frac{r_{s}}{l}\right)^{2}\left[\pi\left(l-2 r_{s}\right)^{2}+2\left(l-r_{s}\right)\left(2 \pi r_{s}+\frac{\pi}{2}+r_{s}\right)\right]
$$

Proof: Since the sensor nodes uniform distribution function from equation (2) can be obtained covering expectations for $E(C)=P\left(\Omega_{I}\right) E\left(C_{\Omega_{I}}\right)+P\left(\Omega_{I I}\right) E\left(C_{\Omega_{I}}\right)$, the formula (3), (4), (5) into equation (2) can be obtained:

$$
\begin{aligned}
E(C) & =P\left(\Omega_{I}\right) E\left(C_{\Omega_{I}}\right)+P\left(\Omega_{I I}\right) E\left(C_{\Omega_{I I}}\right) \\
& =\frac{1}{l^{2}}\left[\pi r_{s}^{2}\left(l-2 r_{s}\right)^{2}\right]+\frac{1}{l^{2}}\left[2 r_{s}^{2} \int_{0}^{l-r_{s}}(2 \pi-\theta+\sin \theta) d x \int_{0}^{r_{s}} d y\right] \\
& =\left(\frac{r_{s}}{l}\right)^{2}\left[\pi\left(l-2 r_{s}\right)^{2}+2\left(l-r_{s}\right)\left(2 \pi r_{s}+\frac{\pi}{2}+r_{s}\right)\right]
\end{aligned}
$$

Theorem 2: Monitoring of the square area for the least number of nodes is effective coverage: $N=\ln (1-\varepsilon) \ln \left(1-\frac{k \pi r^{2}}{l^{2}}\right)^{-1}$, where $K$ is a dimensionless ratio.

Proof: Given a very small amount $\varepsilon(\varepsilon=0.00005)$, to calculate the conclusion of Theorem 1 as a standard, non-zero for any node energy sensor node, its coverage is greater than $\varepsilon$, namely:

$$
P(A)=1-\prod_{1 \leq i \leq k}(1-P(A)) \geq \varepsilon
$$

Then: $1-(1-p)^{N} \geq \varepsilon$, the equation (7) into equation (5) and (6) can be obtained:

$$
1-\left(1-\frac{k \pi r^{2}}{l^{2}}\right)^{N} \geq \varepsilon
$$

Calculated:

$$
N \leq \ln (1-\varepsilon) \ln \left(1-\frac{k \pi r^{2}}{l^{2}}\right)^{-1}
$$

When the left and right sides of the equation are equal, $N$ is the minimum number of sensor nodes.

\subsection{Network Connectivity}

The definition 4 shows of $d_{\min }(G)>0$ is the necessary condition for communicating the communication diagram of $G$, but not sufficient condition. This gives a $P\left(C_{n}\right) \leqslant P$ $\left(d_{m i n}(G)>0\right)$, in practical work, such as node density can not know the case, the lower limit of the $P\left(C_{n}\right)$ has a greater significance[14]. Network communication graph is assumed to exist only in isolation from empty graph nodes begin to increase node communication radius $r_{t}$ while the corresponding communication node corresponding increase in the number of links; obtain minimum node when the node degree $k$, this figure has become a $k$-connected graph. If, after the network diagram, remove any $k$ - 1 nodes, but this figure is still connected, we call $k$ connected graph. Thus, for $k=1$, as long as its communication radius $r_{t}$ large enough so that $d_{\min }(G)>0$, then the network has become a 
communication network, namely: $P\left(C_{n}\right)=P\left(d_{\min }(G)>0\right)$ Since the distribution node are independent, according to the formula (1) can be obtained, for any node $p$ and $(P \in G)$ limits node connectivity rate: Namely the minimum value $\lim _{n \rightarrow \infty}\left[1-\left(1-\frac{S_{p}}{\Omega}\right)^{n}\right]=1-e^{-n S_{p} / \Omega}$ available node $G$ is:

$$
P\left(d_{\text {min }}(G)>0\right)=\prod_{\forall p \in G}\left(1-e^{-n S_{p} / \Omega}\right)
$$

Effective communication had area where $S_{p}$ represents a node $p$, and without considering the influence of the boundary factor $P\left(C_{n}\right)=P\left(d_{\min }(G)>0\right)=\left(1-e^{-n \pi r_{t}^{2} / l^{2}}\right)^{n}$. In fact, when the node is in zone $p, S_{p}$ is equal to the value of the node $p$ is the communication area of a circle, when the node $p$ in zone $I I$, the value of $S_{p}$ is equal to the circumference of the communication area by subtracting the area of the actuate $S_{A C B D}$, thereby obtaining:

$$
P\left(d_{\min }(G)>0\right)=\left(1-e^{-n \pi r_{t}^{2} / l^{2}}\right)^{4 n r_{t}\left(l-r_{t}\right) / l^{2}} \times \prod_{\forall p \in \Omega_{\| I}}\left(1-e^{-n S_{p} / \Omega}\right)
$$

Assuming that node $q$ in the border area of a square deployment, $S_{p}>S_{q}$ point $S_{q}=\frac{1}{2} \pi r_{t}^{2}$, so get:

$$
\prod_{\forall p \in \Omega_{I I}}\left(1-e^{-n S_{p} / \Omega}\right)>\prod_{\forall p \in \Omega_{I I}}\left(1-e^{-n S_{q} / \Omega}\right)=\left(1-e^{-\frac{1}{2} n \pi r_{l}^{2} / l^{2}}\right)^{4 n r_{t}\left(l-r_{t}\right) / l^{2}}
$$

Theorem 3: Given a length 1 of the side square area, the number of nodes is $n$, the communication node is the radius $r_{t}$, in consideration of the boundary elements of the case, the lower limit of the probability of the communication network as follows:

$$
P\left(C_{n}\right)>\left[\left(1-e^{-n \pi r_{t}^{2} / l^{2}}\right)\left(1-e^{-\frac{1}{2} n \pi r_{t}^{2} / l^{2}}\right)\right]^{4 n n_{t}\left(l-r_{n}\right) / l^{2}}
$$

Proof: The formula (10), (11), (12) can be substituted for confirmation. Given $l=100 \mathrm{~m}$, $r_{t}=40 \mathrm{~m}$, according to the probability of obtaining network connectivity theorem 3 , in consideration of the border area, deploying 124 nodes greater than $99.9 \%$, which reached a fully connected network.

Theorem 4: In a monitoring area, located sensor nodes coverage $p$, complete coverage of the target node for the first time after its first cover the expected value is $E(X)=[1-(1-$ $p)^{N} \mathrm{pp}^{-1}, N$ is the number of the target node metastasis.

Proof: Let $X$ be the number of sensor nodes first complete coverage of the target when the target node metastasis, the range of possible values of $X$ is $X \in[1,2,3 \ldots N]$, when $X=k$ when and $1 \leqslant k \leqslant N-1$ is expressed as $N-1$ and before the target node is not covered, so the distribution density function of $X$ is:

$$
P(X=k)=\left\{\begin{array}{l}
p(1-p)^{k-1}, k=1,2,3 \cdots N-1 \\
(1-p)^{N-1}, k=N
\end{array}\right.
$$

Definition, according to expectations:

$$
E(X)=\sum_{k=1}^{N-1} k p(1-p)^{k-1}+N(1-p)^{N-1}
$$


So that $q=1-p, S=\sum_{k=1}^{N-1} k(1-p)^{k-1}$, namely: $S=\sum_{k=1}^{N-1} k q^{k-1}$ multiplied by $q$ in the left ends of the formula, $q S=\sum_{k=1}^{N-1} k q^{k}$ can be obtained, namely:

$$
S=\frac{1-q^{N-1}}{(1-q)^{2}}-\frac{(N-1) q^{N-1}}{1-q}=\frac{1-(1-p)^{N}}{p^{2}}-\frac{N(1-p)^{N-1}}{p}
$$

Equation (16) into equation (15) can be obtained:

$$
\begin{aligned}
E(X) & =p\left(\frac{1-(1-p)^{N-1}}{p^{2}}-\frac{(N-1)(1-p)^{N-1}}{p}\right)+N(1-p)^{N-1} \\
& =\frac{1-(1-p)^{N-1}}{p}-(N-1)(1-p)^{N-1}+N(1-p)^{N-1} \\
& =\frac{1-(1-p)^{N-1}+p(1-p)^{N-1}}{p}=\left[1-(1-p)^{N}\right] p^{-1}
\end{aligned}
$$

That is: $E(X)=\left[-(1-p)^{N}\right] \mathrm{p}^{-1}$.

In order to achieve network coverage and connectivity of different rate, you need to deploy the relationship between the number of nodes as shown in Table 2: In this table you can find the number of values corresponding to deploy nodes. When the network coverage and connectivity rate greater (typically $\geqq 99 \%$ ) when, in this case can be considered completely covered with network connectivity. When the network connectivity rate $P\left(C_{n}\right)$ network coverage equal to $E\left(S_{n}\right)$ when you can see from Table 2, get network coverage and network connectivity rate of the same value, the need to deploy more nodes, as shown in Table 2 :

Table 2. Deployment of Network Coverage and Connectivity Required

\begin{tabular}{ccccccc}
\hline & $\begin{array}{c}l=100 \mathrm{~m} \\
\text { coverage }\end{array}$ & $\begin{array}{c}l=100 \mathrm{~m} \\
\text { connect }\end{array}$ & $\begin{array}{c}l=200 \mathrm{~m} \\
\text { coverage }\end{array}$ & $\begin{array}{c}l=200 \mathrm{~m} \\
\text { connect }\end{array}$ & $\begin{array}{c}l=300 \mathrm{~m} \\
\text { coverage }\end{array}$ & $\begin{array}{c}l=300 \mathrm{~m} \\
\text { connect }\end{array}$ \\
\hline $10 \%$ & 1 & 5 & 4 & 43 & 8 & 124 \\
$20 \%$ & 2 & 6 & 7 & 50 & 16 & 132 \\
$30 \%$ & 3 & 8 & 11 & 56 & 25 & 144 \\
$40 \%$ & 4 & 10 & 15 & 61 & 36 & 152 \\
$50 \%$ & 5 & 13 & 21 & 68 & 48 & 166 \\
$60 \%$ & 7 & 17 & 27 & 75 & 64 & 184 \\
$70 \%$ & 9 & 20 & 36 & 82 & 82 & 205 \\
$80 \%$ & 11 & 25 & 48 & 91 & 110 & 242 \\
$90 \%$ & 16 & 30 & 68 & 106 & 158 & 294 \\
$100 \%$ & 33 & 43 & 136 & 151 & 314 & 350 \\
\hline
\end{tabular}

\section{Problem Definition and Network Models}

In order to better evaluate network performance, using MATLAB6.5 simulation experiments. By changing the area of coverage, network coverage and connectivity to achieve different scale, and thus better assess model performance under different scale, mainly in the case of the number of different coverage and achieve network connectivity rate of the need to deploy a minimum of nodes, each simulation results are the average of 50 times. Graph showing changes in the node coverage under different network size, shown in Figure 2: 


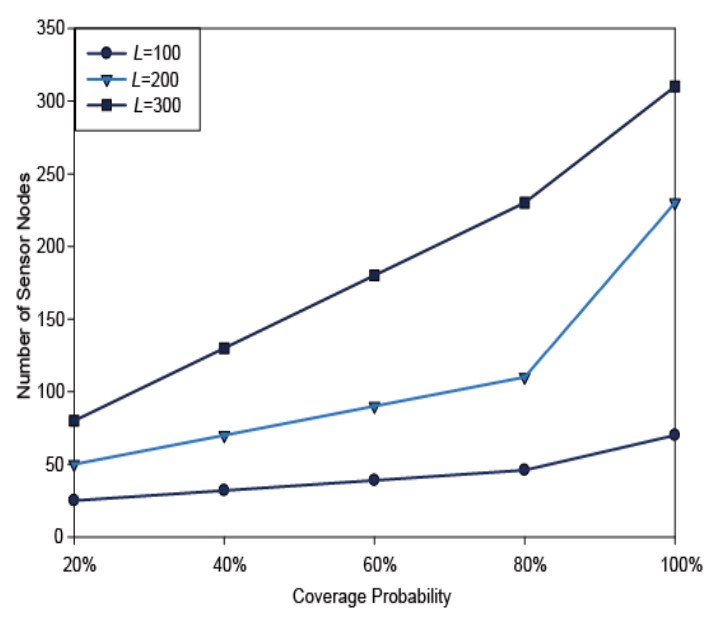

Figure 2. Node Coverage under Different Network Size Curve

Figure 2 shows the size of the network at different nodes to achieve coverage under different number of sensor nodes need to be deployed in Fig2. Can be obtained from the chart with the expansion of network size, when the network coverage to meet certain requirements, the number of nodes required for de ployment will increase, and the higher the coverage of the network, increasing the number of nodes need to be deployed more fast, so that the target node of interest to achieve complete coverage.

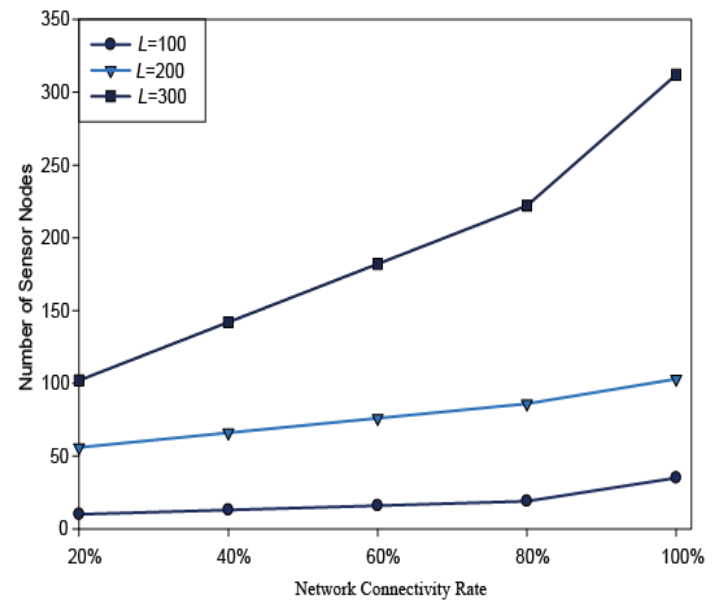

Figure 3. The Size of Network under Different Node Connectivity Rate Curve

Figure 3 shows the size of the network at different network connectivity rate achieved under different number of nodes need to be deployed sensor diagram. With the expansion of the network to achieve certain network connectivity rate, the number of nodes need to be deployed will increase, when the high connectivity rate, increase the number of nodes need to be deployed rapidly.

In considering whether the boundary conditions affect the rate of coverage and connectivity. Figure 4 and Figure 5 shows the influence of the presence or absence of boundary conditions, the amount of contrast in the same network size $l=300 \mathrm{~m}$ coverage and connectivity rate sensor nodes need to be deployed. We can see from the chart, with increasing network coverage and connectivity rate, a significant increase in the number of nodes needed; when the coverage and network 
connectivity rate increases, the impact on the boundary becomes smaller, and finally tends to equilibrium.

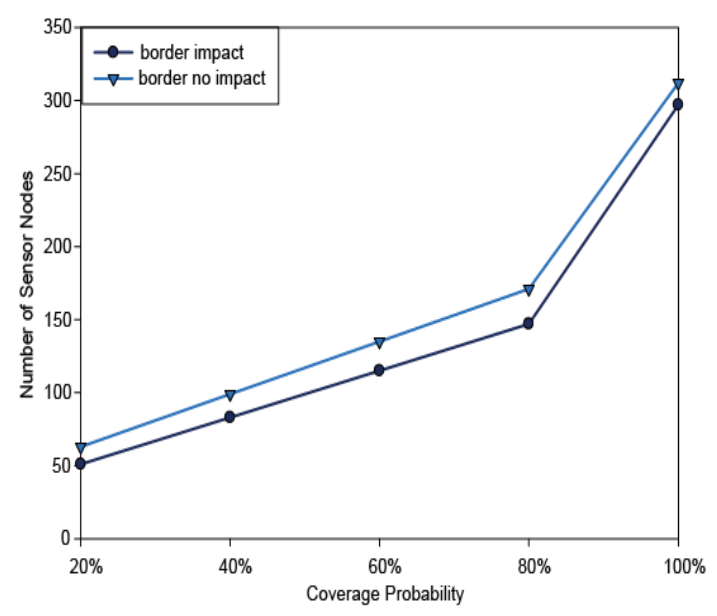

Figure 4. Impact of Network Coverage under the Boundary Curve

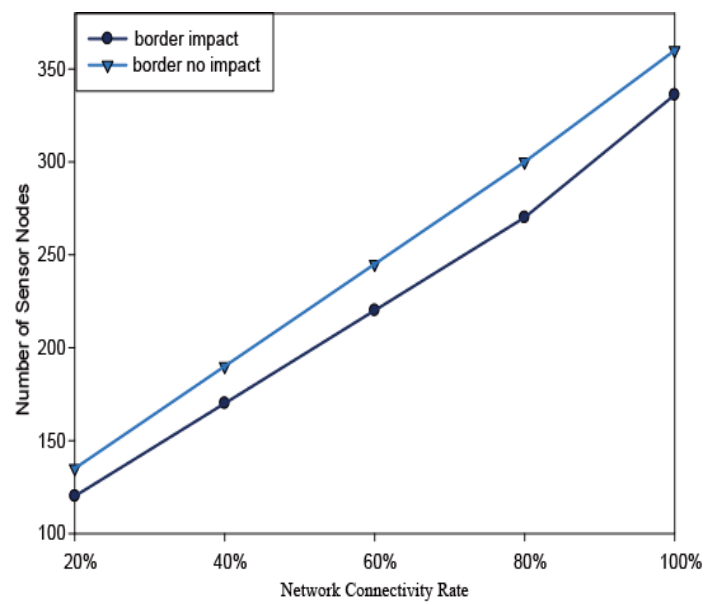

Figure 5. Network Connectivity Lower Boundary Curves

Figures 4 and 5 the presence or absence of reaction are considered under the influence of the boundary conditions for the realization of different rates of coverage and connectivity to deploy the number of map nodes. Compared to consider the boundary under the influence of a slight increase in the number of nodes deployed, the number of nodes increases, the density between the nodes will also become larger, the impact on the border decreased.

\section{Conclusion}

In this paper, for wireless sensor networks network coverage and connectivity issues randomly deployed nodes, gives the sensor node and the destination node associated with the relational model [15]. In considering the impact of the boundary conditions, we propose a target associated with coverage and connectivity control methods. It can simplify the computational complexity of network coverage and connectivity, and improve the efficiency of the algorithm to achieve a certain network to meet the requirements of coverage and connectivity rate, the more accurately the deployment of nodes needed to solve a number of values [16]. Finally, the simulation results show the effectiveness of the correctness of the 
theory and algorithms for solving of which certain specific methods scalability and extensibility can be used to solve the problem of multiple heterogeneous connectivity coverage under the form.

\section{Acknowledgements}

The authors wish to thank Science of Technology Research of Foundation Project of Henan Province Education Department under Grant Nos.2014B520099; Natural Science and Technology Research of Foundation Project of Henan Province Department of Science under Grant Nos. 142102210471, 142102210063; Natural Science and Technology Research of Foundation Project of Luoyang Department of Science under Grant Nos. 1401037A.

\section{References}

[1] B. Wang, K. C. Chua, V. Srinivasan, W. Wang, "Information coverage in randomly deployed wireless sensor networks", IEEE Transactions on Wireless Communications, (2007).

[2] Y. C. Tseng, P. Y. Chen and W. T. Chen, "K-Angle object coverage problem in a wireless sensor network", Sensors Journal, vol.12, no.3408, (2012).

[3] Y. Xiao, H. Chen, K. Wu, B. Sun, Y. Zhang, X. Y. Sun and C. Liu, "Coverage and detection of a randomized scheduling algorithm in wireless sensor networks", vol.4, no. 507, (2010).

[4] H. Z. Wan, F. Z. Meng, Z. Z. Li, "Energy efficient coverage conserving protocol for wireless sensor networks", Journal of Software, vol.12, no.3124, (2010).

[5] X. He X, X. L. Gui and J. An, "A distributed area coverage algorithm based on delayed awakening in wireless sensor networks",Journal of Computer Research and Development, vol.5, no.786, (2011).

[6] R. Tahiry and S. R. David, "Connectivity preservation and coverage schemes for wireless sensor networks", IEEE Transactions on Automatic Control, vol.10, no. 2418, (2011).

[7] H. M. Ammari and S. K. Das, "Centralized and clustered k-coverage protocols for wireless sensor networks", IEEE Transactions on Computers, vol.6, no.118, (2012).

[8] J. Z. Du, K. Wang and D. Guo, "Maximizing the lifetime of k-discrete barrier coverage using mobile sensors", IEEE Sensors Journal, vol.12, no.4690, (2013).

[9] M. Cardei, D. Z. Du, "Improving wireless sensor network lifetime through power aware organization", Wireless Networks, vol.3, no.333, (2005).

[10] S. H. Yang, F. Dai, M. Cardei, J. Wu and F. Patterson, "On connected multiple point coverage in wireless sensor networks", International Journal of Wireless Information Networks vol.4, no.289, (2006).

[11] H. Liu, P. Wan, C. W. Yi, X. H. Jia and P. Niki, "Maximal lifetime scheduling in sensor surveillance networks", IEEE Transactions on Computers, vol.11, no.557, (2011).

[12] S. Mini, S. K. Udgata and S. L. Sabat, "Sensor deployment and scheduling for target coverage problem in wireless sensor network", IEEE Sensor Journal, vol.3, no.636 (2014).

[13] Y. Jin, J. Y. Jo, L. Wang, Y. Kim and X. Z. Yang, "ECCRA: An energy-efficient coverage and connectivity preserving routing algorithm under border effects in wireless sensor networks", Computer Communications, vol.10, no.2398, (2008).

[14] J. Chen, L. Zhang and Y. Kuo, "Coverage-enhancing algorithm based on overlap-sense ratio in wireless multimedia sensor networks", IEEE Sensors Journal, vol.6, no. 2077, (2013).

[15] S. Zeyu, W. Weiguo and W. Huanzhao, "A Novel Coverage Algorithm Based on Event-ProbabilityDriven Mechanism in Wireless Sensor Network", Journal on Wireless Communications and Networking, vol.4, no.1, (2014).

[16] S. Zeyu, W. Weiguo and W. Huanzhao, "An Optimized Strategy Coverage Control Algorithm for WSN", International Journal of Distributed Sensor Networks, vol.6, no.1, (2014). 


\section{Authors}

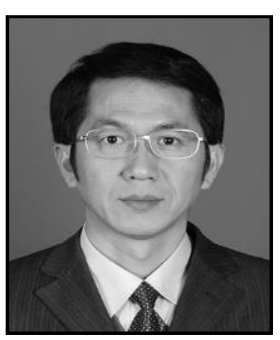

Yunxing Shu, he was born in 1962 in Nantong, Jiangsu province, in 1994 graduated from Tsinghua University Computer College with a master's degree in engineering, 2008, graduated from Wuhan University of science and engineering $\mathrm{PhD}$, professor of Luoyang institute of technology. Main research interests is computer modeling and simulation, complex networks and parallel computing.

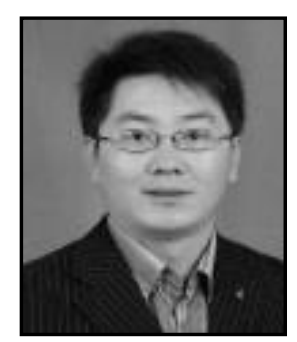

Zeyu Sun, he was born in 1977 in Changchun city, Jilin province, in 2010 graduated from Lanzhou university, Master of Science; xi 'an Jiaotong university study for $\mathrm{PhD}$ degree at present. He is a lecturer in Luoyang institute of technology of computer and information engineering, and a member of China computer society, and IEEE Student. The main research interest is wireless sensor networks, parallel computing and Internet of things.

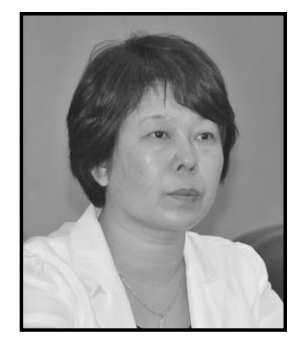

Bo Ge, she was born in 1962 in Fengcheng city, Liaoning province, professor. In 1983, graduated from Wuhan Polytechnic University, bachelor's degree; in 1989, graduated from Beijing Aerospace Day University, a master's degree; in 2007, graduated from Wuhan University of Technology and engineering $\mathrm{PhD}$, The main research interest is wireless sensor networks, parallel computing and Internet of things. 